\title{
The Effect of Nicotine and its Interaction with Ethanol on Biochemical Parameters, Oxidative Damage and Histological Changes in the Rat's Liver
}

\author{
Hanène Dhouib ${ }^{1}$; Manel Jallouli ${ }^{1}$; Monia Draief ${ }^{2}$; Saloua El-Fazaa ${ }^{1 *}$, Saadia \\ Bouraoui $^{2 *}$ \\ 1. Physiology Laboratory, Department of Biology, Faculty of Sciences, University Campus, 2092 Manar II, \\ Tunis, Tunisia \\ 2. Department of Anatomopathology, El Mongi Slim Hospital, la Marsa, Tunis, Tunisia.
}

\begin{abstract}
In the present study we have evaluated the effect of chronic nicotine exposure and ethanol supplementation on biochemical parameters and oxidative stress in serum and liver of rats, respectively. We also performed histological analysis in liver. The nicotine-treated rats showed a weak effect on the activities of markers of liver function and a significant increase in the level of lactate dehydrogenase (LDH). The combined exposure significantly increased the level of aspartate aminotransferase (AST), alanine aminotransferase (ALT), alkaline phosphatase $(A L P)$, and lactate dehydrogenase $(L D H)$. Moreover, the results showed that nicotine significantly increased lipid peroxidation, superoxide dismutase (SOD) activity and enhanced catalase (CAT) activity in liver. The combination of nicotine plus ethanol revealed the same effect in the level of lipid peroxidation and SOD activity, whereas the level of CAT activity was significantly raised. Histological studies showed morphological alterations in liver rat in both treated groups compared to control rats. Also, comparing the results of the nicotine-group to nicotine ethanol-group, we concluded that nicotine had prejudicial effects of less intensity than the association of nicotine and ethanol. These finding suggest that chronic nicotine administration provoked harmful effects to the liver; this hepatotoxicity can be enhanced by supplementation of ethanol and this association certainly increase the risk to develop liver diseases. Finally, the liver toxicity induced by nicotine alone or supplemented with ethanol was revealed by increasing both lipid peroxidation and oxidative stress in the liver, disturbance of indicators of liver function, enhance in the level of LDH and change of histological parameters.
\end{abstract}

Keywords: Nicotine exposure, Ethanol supplementation, Liver, Oxidative stress, Hepatotoxicity, Rats.

\section{Introduction}

Tobacco is the most widely used drug in the word and the greatest causes of illness and premature death in developed and developing countries. Epidemiological studies have shown a relationship between smoking and increased risks of cardiovascular disorders, lung cancer and pulmonary diseases. ${ }^{1-4}$ Moreover, it has been shown that cigarette smoking may accelerate the progression of renal, pulmonary, and cardiac fibrosis. ${ }^{5-7}$ The detrimental effects of smoking have beenextensively investigated by studies of direct administration of nicotine, a major pharmacologically active component of tobacco smoke, ${ }^{8}$ in animal and in a variety of cell systems. The predominant effects of nicotine in the whole intact animal or human consist of an increase inheart rate (10 to 20 beats $/ \mathrm{min}$ ), blood pressure (5 to $10 \mathrm{mmHg}$ ), release of catecholamines and free fatty acids and mobilization of blood sugar. ${ }^{9-11}$

Numerous experimental and clinical evidences have supported the key role of oxidative stress in the pathogenesis of organ disorders after nicotine exposure. ${ }^{12-15}$ Indeed, nicotine significantly increased oxidative stress by enhancing the generation of reactive oxygen species, ${ }^{16}$ and lipid peroxidation. ${ }^{17}$ In addition, nicotine induced a depletion of antioxidant defense systems through the reduction of catalase and superoxide dismutase activities and level of glutathione peroxidase. ${ }^{18}$ Nicotine, once absorbed, is mainly metabolized by the liver to a number of major and minor metabolites. ${ }^{19,20}$ The major metabolite is cotinine, the primary product of the Coxidation pathway of nicotine biotransformation has been used as a marker for nicotine intake. ${ }^{21}$

Seeing that the liver is the major site of nicotine metabolism, it has been considered highly susceptible for the oxidative stress associated with the toxicity of nicotine.In fact, many epidemiological studies have shown an association between smoking and accelerated progression of liver fibrosis in patients with a variety of chronic liver diseases such as primary biliary cirrhosis and chronic hepatitis $C^{22,23}$ The experimental models of Azzalini et al ${ }^{24}$ have shown that smoking caused oxidative stress and exacerbated the severity of nonalcoholic fatty liver disease in obese rats.Moreover, nicotine from heavy smoking increased the risk of developing hepatocellular carcinoma $(\mathrm{HCC})^{25,26}$ and liver cirrhosis. ${ }^{27,28}$ Yuen and colleagues ${ }^{29}$ reported that nicotine administration at a concentration similar to those attained by cigarette smoking was hepatotoxic. ${ }^{30}$ 
In this respect, Husain and coworkers demonstrated that ethanol supplementedto nicotine treatment might augment hepatotoxicity and oxidative damage in liver of nicotine treated rats. ${ }^{31}$ In fact, numerous investigations have revealed that nicotine and ethanol as individual source for ROS production ${ }^{32,33}$ and oxidative damage in important components of the cellular machinery. ${ }^{34}$ In addition, when ethanol was metabolized in the liver, reactive species were generated and lipid peroxidation wasenhanced. ${ }^{35,36}$

Seeing the increasing rate of co-use and co-abuse of smoking cigarette and drinking alcohol, ${ }^{37,38}$ it was imperative to investigate the effects of nicotine administration and its interactive effects with ethanol supplementation.

The aim of this study was to evaluate the hepatotoxicity of chronic nicotine exposure and to compare the effect of nicotine alone to its combination with ethanol. We investigated serum biochemical parameters, such as ALP, ALT, AST and LDH.Oxidative parameters, namely MDA, the activities of the antioxidant enzymes catalase and superoxide dismutase in the liver of rats, were evaluated. We also performed histological analysis in the liver of treated rats.

\section{Chemicals}

\section{Materials and methods}

Nicotine hydrogen bitartrate was obtained from Sigma Chemical Company, St. Louis, Missouri, USA. Absolute ethanol (99.5\%) was purchased from Carlo Erba reagent (France). All other chemicals and reagents used were of analytical grade.

\section{Animals}

Male albino Wistar rats, of 8 weeks of age and weighing about 120-150 g, were purchased from SIPHAT (Tunis, Tunisia). Animals were housed 2 to a polypropylene cage and provided with food and water ad libitum. Before beginning the experiment, all animals were acclimated for 1 week under well-controlled conditions of temperature $\left(22 \pm 2^{\circ} \mathrm{C}\right)$, relative humidity $(70 \pm 4 \%)$, and a 12/12 h light-dark cycle with 07:3019:30 h being light phase. Animals were fed standard pellet diet (SISCO, Sfax, Tunisia). Procedures involving the animals and their care were conformed to the institutional guidelines, in compliance with national and international laws and guidelines for use of animals in biomedical research (Giles AR., 1987). ${ }^{39}$

\section{Experimental design}

Rats were divided into three groups of eight animals each and treated for 6 weeks as follows: (1) Control group of animals received normal saline by subcutaneous injection daily;(2) nicotine group of animals received nicotine at a dose of $2.5 \mathrm{mg} / \mathrm{kg}$ by subcutaneous injection daily; and (3) nicotine plus ethanol group of animals received $2.5 \mathrm{mg} / \mathrm{kg}$ of nicotine in ethanol $25 \%$ at a dose de $2 \mathrm{~g} / \mathrm{kg}$ by subcutaneous injection daily. Rats in all groups were killed by decapitation $24 \mathrm{~h}$ after the last injections. Target tissues (lung, kidney and liver) were excised from animals at the time of sacrifice, cleared off blood and immediately transferred to ice-cold containers containing $0.9 \%$ sodium chloride for various estimations.

\section{Preparation of serum and tissue extracts}

Blood samples were collected from all groups of rats into centrifuge tubes. Blood was allowed to clot at room temperature for about $30 \mathrm{~min}$. Serum was separated by centrifugation at $3000 \mathrm{rpm}$ for $10 \mathrm{~min}$ and stored at $-80^{\circ} \mathrm{C}$ in aliquots until analysis. Livers were excised immediately, washed with ice-gold physiologic saline solution $(0.9 \%)$, blotted dry and weighed. A portion of livers were homogenized in 10 volumes $(1: 10$; w/v) of ice-cold $10 \mathrm{mM}$ phosphate buffered saline (PBS, $\mathrm{pH}$ 7.4) in an Ultra Turrax tissue homogenizer for 30s. Homogenates were centrifuged at $10.000 \mathrm{rpm}$ for $10 \mathrm{~min}$ at $4^{\circ} \mathrm{C}$. Thesupernatant was immediately pipetted into clean centrifuge tubes and stored in aliquots $\left(-80^{\circ} \mathrm{C}\right)$ until analysis.

\section{Haematological parameters \\ 5.1. Hematocrit content}

A hematocrit measurement was carried out in capillary tubes centrifuged with HEMATOCRIT 20 Hettich for $15 \mathrm{~min}$ at $1000 \mathrm{rpm}$.

\subsection{Hemoglobin concentration}

Hemoglobin concentrations in whole blood were spectrophotometrically analyzed at $540 \mathrm{~nm}$ by the cyanomethemoglobin method. ${ }^{40}$ Blood samples were mixed with $5 \mathrm{ml}$ Drabkin's solution $(0.1 \%$ sodiumbicarbonate, $0.005 \%$ potassium cyanide and $0.02 \%$ potassiumferricyanide) for hemoglobin determination. Hb standard was purchased from Sigma.

\section{Biochemical parameters}


Biochemical parameters of liver function i.e., aspartate aminotransferase (AST), alanine aminotransferase (ALT), alkaline phosphatase (ALP), and lactate dehydrogenase (LDH) activities were estimated in serum by using diagnostic kits supplied by Biomaghreb Laboratories (Tunis, Tunisia).

\section{Oxidative stress parameters}

\subsection{Determination of liver lipid peroxidation}

The level of lipid peroxidation products in liver was measured according to the method of Yagi K. ${ }^{41}$ The level of malondialdehyde (MDA), a marker for lipid peroxidation, was assayed by monitoring thiobarbituric reactive substances (TBARS) formation. Briefly, $500 \mu 1$ of liver homogenate was added to $200 \mu 1$ of phosphate buffered saline (PBS, 10Mm, pH 7.4) and $500 \mu \mathrm{l}$ of heat trichloroacetic acid- butylatedhydroxytoluene (20\% TCA, $1 \%$ BHT) solution. The resultant was thoroughly mixed and centrifuged at $3000 \mathrm{rpm}$ for $10 \mathrm{~min} 4^{\circ} \mathrm{C}$. To $800 \mu \mathrm{l}$ of supernatant, $160 \mu \mathrm{l}$ of $0.6 \mathrm{M} \mathrm{HCL}$ and $640 \mu \mathrm{l}$ of $1.73 \%$ thiobarbiturique acid (TBA) dissolved in Tris, were added. This suspension was mixed and heated in a boiling water bath for 15 minutes. After cooling, the thiobarbituric reactive substances (TBARS) were measured in supernatant at $530 \mathrm{~nm}$ against a blank containing all reagents except the tissue homogenate. The concentration of MDA was calculated by the extinction coefficient of MDA-TBA complex $\left(1.56 \times 10^{5} \mathrm{~L} \mathrm{~mol}^{-1} \mathrm{~cm}^{-1}\right)$ and expressed in nanomoles per milligram of protein.

\subsection{Determination of liver SOD activity}

Superoxide dismutase activity (SOD) was determined at room temperature by using a slightly modified version of Misra and Fridovichmethod. ${ }^{42}$ Five microliters of $10 \%$ liver homogenate was added to $1965 \mu 1$ (0.05M, pH 10.2, $0.1 \mathrm{mM}$ EDTA) of sodium carbonate buffer plus ten microliters of bovine catalase. Twenty microliters of $30 \mathrm{mM}$ epinephrine (dissolved in $0.05 \%$ acetic acid) was added to the mixture to start the reaction. Superoxide dismutase activity was measured at $480 \mathrm{~nm}$ for $5 \mathrm{~min}$ on a spectrophotometer. Activity was expressed as the amount of the enzyme that inhibits the oxidation of epinephrine by $50 \%$, which is equal to $1 \mathrm{U}$ per milligram of protein.

\subsection{Determination of liver CAT activity}

Catalase (CAT) activity was assayed at room temperature according to the method of Aebi slightly modified. ${ }^{43}$ Twenty microliters of $10 \%$ liver homogenate was added to a cuvette containing $780 \mu 1(0.05 \mathrm{M}, \mathrm{pH}$ 7, $0.1 \mathrm{mM}$ EDTA) of sodium phosphate buffer, and $200 \mu 1$ of hydrogen peroxide $\left(\mathrm{H}_{2} \mathrm{O}_{2}, 3 \%\right)$ was added to start the reaction. Catalase activity was measured at $240 \mathrm{~nm}$ for $3 \mathrm{~min}$ with the use a spectrophotometer. The molar extinction coefficient of $43.6 \mathrm{M}^{-1} \mathrm{~cm}^{-1}$ was used to determine CAT activity. One unit of activity is equal to the $\mu$ moles of $\mathrm{H}_{2} \mathrm{O}_{2}$ degraded per minute per milligram of protein.

\section{Protein assay}

Protein concentrations in the serum and the homogenate of liver were determined by the method of Bradford ${ }^{44}$ using bovine serum albumin as standard.

\section{Histological examination}

For histological examination, fragments from central portion, left and right lobes of the liver were removed, fixed in alcohol, formalin, and acetic acid (AFA) for $24 \mathrm{~h}$, and then embedded in paraffin. A setof 5$\mu \mathrm{m}$-thick sections was placed in glass slides and stained with hematoxylin and eosin for morphological analysis. $^{45}$

\section{Statistical analysis}

All results are expressed as mean \pm SEM. Statistical significance of the difference between group means was performed by one-way ANOVA followed by Student's t-test. Differences with $\mathrm{p}<0.05$ were considered to be statistically significant.

\section{General characteristics of rats}

\section{Results}

As shown in table.1, chronic nicotine administration had no effect on the absolute $(\mathrm{g})$ and relative (g/100g b.w) weight of the liver. However, when ethanol was supplemented with nicotine, a significant increase in weight of liver rat was observed compared to the control $(\mathrm{p}<0.05)$.

As shown in Table.2, chronic nicotine administration induced a significant increase in hematocrit content and hemoglobin. However, when ethanol was supplemented with nicotine, there was no significant difference in hematological parameters compared to the control group. 
Table1. Effects of chronic nicotine administration $(2.5 \mathrm{mg} / \mathrm{kg}$ bw, s.c) alone or associated with ethanol on the body weight, absolute and relative liver weight in rats

\begin{tabular}{lrrr}
\hline Parameter & Control $(\mathrm{n}=8)$ & Nicotine $(\mathrm{n}=8)$ & Nicotine/Ethanol $(\mathrm{n}=8)$ \\
\hline Body weight $(\mathrm{g})$ & $231.38 \pm 5.59$ & $218.89 \pm 5.21$ & $228.00 \pm 4.87$ \\
Liver weight $(\mathrm{g})$ & $7.33 \pm 0.18$ & $6.92 \pm 0.18^{\mathrm{c}}$ & $7.63 \pm 0.17$ \\
Relative liver weight $(\mathrm{g})$ & $3.18 \pm 0.08$ & $3.17 \pm 0.10$ & $3.25 \pm 0.09$ \\
\hline
\end{tabular}

Each value is mean \pm SEM; number of rats in parenthesis.

${ }_{\mathrm{p}}^{\mathrm{p}}<0.05$ nicotine group vs. nicotine-ethanol group.

Table2. Effects of subcutaneous injections of nicotine $(2.5 \mathrm{mg} / \mathrm{kg} \mathrm{bw})$ alone or supplemented with ethanol on hemoglobin and hematocrit

\begin{tabular}{lrcc}
\hline Parameter & Control $(\mathrm{n}=8)$ & Nicotine $(\mathrm{n}=8)$ & Nicotine-Ethanol $(\mathrm{n}=8)$ \\
\hline Hemoglobin $(\mathrm{g} / \mathrm{L})$ & $145.14 \pm 3.12^{\text {aa }}$ & $160.05 \pm 3.02$ & $153.22 \pm 3.70$ \\
Hematocrit $(\%)$ & $47.5 \pm 0.63^{\text {a }}$ & $49.3 \pm 0.33$ & $48.4 \pm 0.46$ \\
Plasma protein $(\mathrm{g} / \mathrm{L})$ & $67.84 \pm 1.20$ & $66.51 \pm 0.88$ & $63.17 \pm 1.06^{\mathrm{b}}$ \\
\hline
\end{tabular}

Each value is mean \pm SEM; number of rats in parenthesis.

${ }^{\mathrm{a}} \mathrm{p}<0.05$ or ${ }^{\mathrm{a} a} \mathrm{p}<0.01$ nicotine group vs. control group.

${ }_{\mathrm{p}}^{\mathrm{p}}<0.05$ nicotine-ethanol group vs. control group.

\section{Biochemical parameters of liver function}

Table3. Biochemical indicators of liver function in serum of control and nicotine-treated rats with or without ethanol supplementation

\begin{tabular}{lrrr}
\hline Parameter & Control $(\mathrm{n}=8)$ & Nicotine $(\mathrm{n}=8)$ & Nicotine-Ethanol $(\mathrm{n}=8)$ \\
\hline ALT (U/L) & $35.00 \pm 1.93$ & $37.19 \pm 0.86^{\mathbf{c}}$ & $44.56 \pm 3.13^{\mathbf{b}}$ \\
AST (U/L) & $103.25 \pm 03.56$ & $108.94 \pm 05.39^{\mathbf{c}}$ & $135.63 \pm 09.07^{\mathbf{b b}}$ \\
ALP (U/L) & $216.86 \pm 10.55$ & $227.56 \pm 13.36$ & $250.53 \pm 06.32^{\mathbf{b b}}$ \\
LDH (U/L) & $746.95 \pm 32.89^{\mathbf{a}}$ & $1022.67 \pm 95.37$ & $1115.09 \pm 82.83^{\mathbf{b b}}$ \\
\hline
\end{tabular}

AST; aspartate aminotransferase, ALT: alanine aminotransferase, ALP: alkaline phosphatase, LDH: lactate dehydrogenase.

Data represent mean \pm S.E.M from eight rats in each group.

${ }^{\mathrm{a}} \mathrm{p}<0.05$ nicotine group vs. control group.

${ }^{\mathrm{b}} \mathrm{p}<0.05$ or ${ }^{\mathrm{bb}} \mathrm{p}<0.01$ nicotine-ethanol group vs. control group.

The effect of chronic nicotine exposure and ethanol supplementation on biochemical indicators of liver function in serum is depicted in Table 3. Compared with the controls, nicotine-treated animals have a slightly increase but no significant ( $\mathrm{p}>0.05$ ) in serum ALT, AST and ALP levels. The increase was 6.25\%, 5.51\% and $4.94 \%$, respectively. However, chronic injection of nicotine increased significantly $(\mathrm{p}<0.05)$ the level of LDH by $36.91 \%$. When nicotine administration was supplemented with ethanol, the activities of these enzymes significantly enhanced $(\mathrm{p}<0.05)$. Thereby, nicotine-ethanol group showed a significant increase in levels of ALT, AST, ALP and LDH by $27.31 \%, 31.36 \%, 15.53 \%$ and $49.28 \%$ respectively, compared to values of control group.

\section{Lipid peroxidation of the liver}

As shown in Fig.1, chronic nicotine administration with or without ethanol supplementation promoted lipid peroxidation; the MDA level in rat liver homogenates was significantly increased in both nicotine and nicotine-ethanol groupsby $189.03 \%(\mathrm{p}<0.05)$ and $204.27 \%(\mathrm{p}<0.01)$ respectively, compared with the values of control group. However no significant difference was registered between nicotine and nicotine-ethanol groups. 


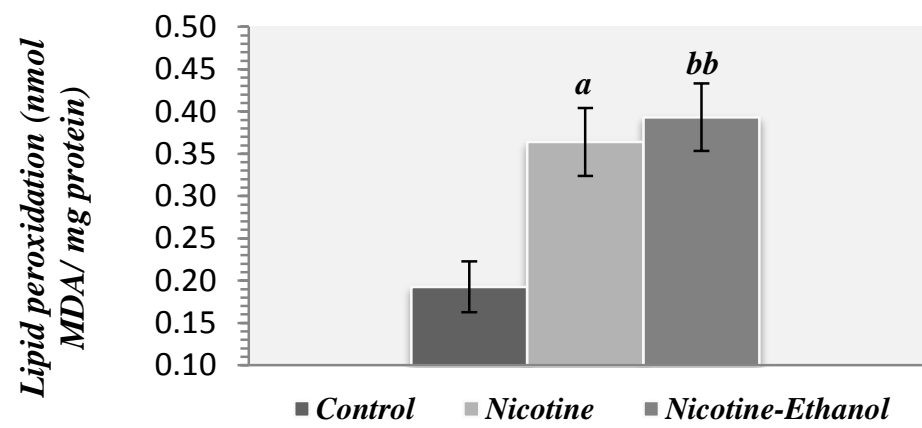

Figure1. Malondialdehyde concentration in livers of rats treated with chronic administration of nicotine (2.5 $\mathrm{mg} / \mathrm{kg}$, s.c.), with or without ethanol supplementation $(2.5 \mathrm{~g} / \mathrm{kg}$, s.c.). Values are expressed as means \pm S.E.M from eight rats in each group.

${ }^{\mathrm{a}} \mathrm{p}<0.05$, when nicotine group was compared with control group.

${ }_{\mathrm{p}}^{\mathrm{b}}<0.05,{ }^{\mathrm{bb}} \mathrm{p}<0.01$, when nicotine-ethanol group was compared with control group.

\section{Activities of liver antioxidant enzymes}

The effect of chronic nicotine administration with or without ethanol supplementation on activities of liver antioxidant enzymes is shown in Figure 2\&3.Compared to controls, nicotine-treated animals showeda significantincrease in liver SOD activity by64.17\%(p < 0.05) and anincrease in liver CAT activity by $41.74 \%$. The combination of nicotine plus ethanol significantly increased liver SOD activity by $82.09 \%(\mathrm{p}<0.01)$, as well as significantly increased liver CAT activity by $64.56 \%(\mathrm{p}<0.05)$ when compared with control values. The results suggest that chronic nicotine administration significantly disrupted the activity of liver SOD; however the enhancedactivity of liver CAT was not significant. When nicotine administration was supplemented with ethanol, we observed a significant increasein activity of both antioxidant enzymes compared with findings of nicotine group.

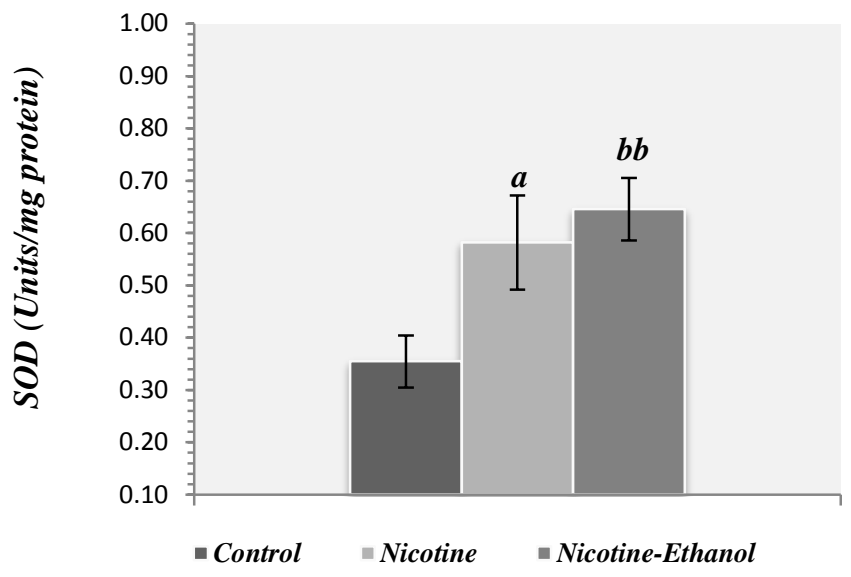

Figure2. Superoxide dismutase activity in livers of rats treated with chronic administration of nicotine (2.5 $\mathrm{mg} / \mathrm{kg}$, s.c. $)$, with or without ethanol supplementation $(2.5 \mathrm{~g} / \mathrm{kg}$, s.c.). Values are expressed as means \pm S.E.M from eight rats in each group.

$\mathrm{U}=$ enzymes required for $50 \%$ inhibition of epinephrine oxidation $/ \mathrm{min} / \mathrm{mg}$ protein.

${ }^{a} \mathrm{p}<0.05$, when nicotine group was compared with control group.

${ }^{b} \mathrm{p}<0.05,{ }^{b b} \mathrm{p}<0.01$ when nicotine-ethanol group was compared with control group 


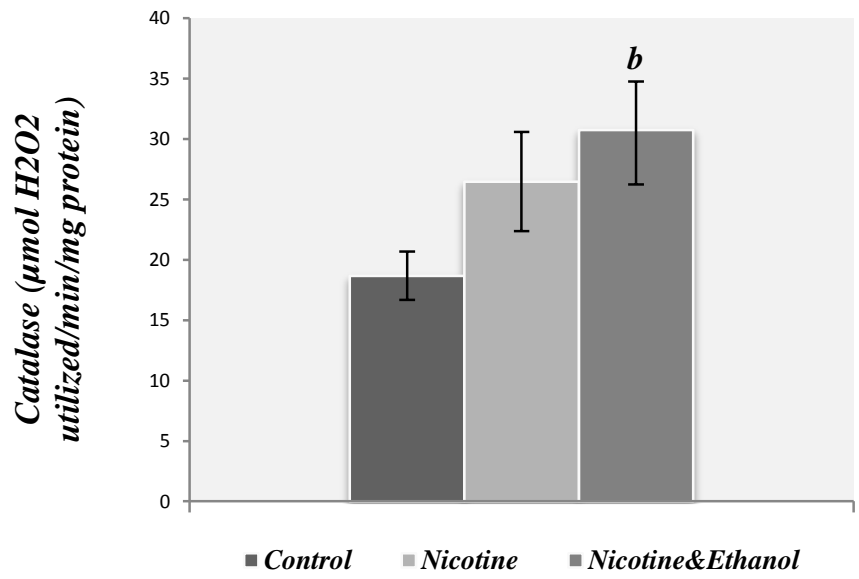

Figure3. Catalase activity in livers of rats treated with chronic administration of nicotine $(2.5 \mathrm{mg} / \mathrm{kg}$, s.c.), with or without ethanol supplementation $(2.5 \mathrm{~g} / \mathrm{kg}$, s.c.). Values are expressed as means \pm S.E.M from eight rats in each group.

${ }^{a} \mathrm{p}<0.05$, when nicotine group was compared with control group.

${ }_{\mathrm{b}}^{\mathrm{p}}<0.05$, when nicotine-ethanol group was compared with control group.

\section{Histological analysis of the liver tissues}

In the liver, histological examination revealed marked tissue damage and changes (figs.4. B \& $\mathrm{C}$ ) when compared with the control (figs.4. A). These changes included loss of trabecular arrangement, congestion of centrilobular veins and sinusoids, and moderate infiltration of lymphocytes. Ethanol supplementation markedly increased the degree of injury, congestion and dilation of veins and diffused microvesicular steatosis.
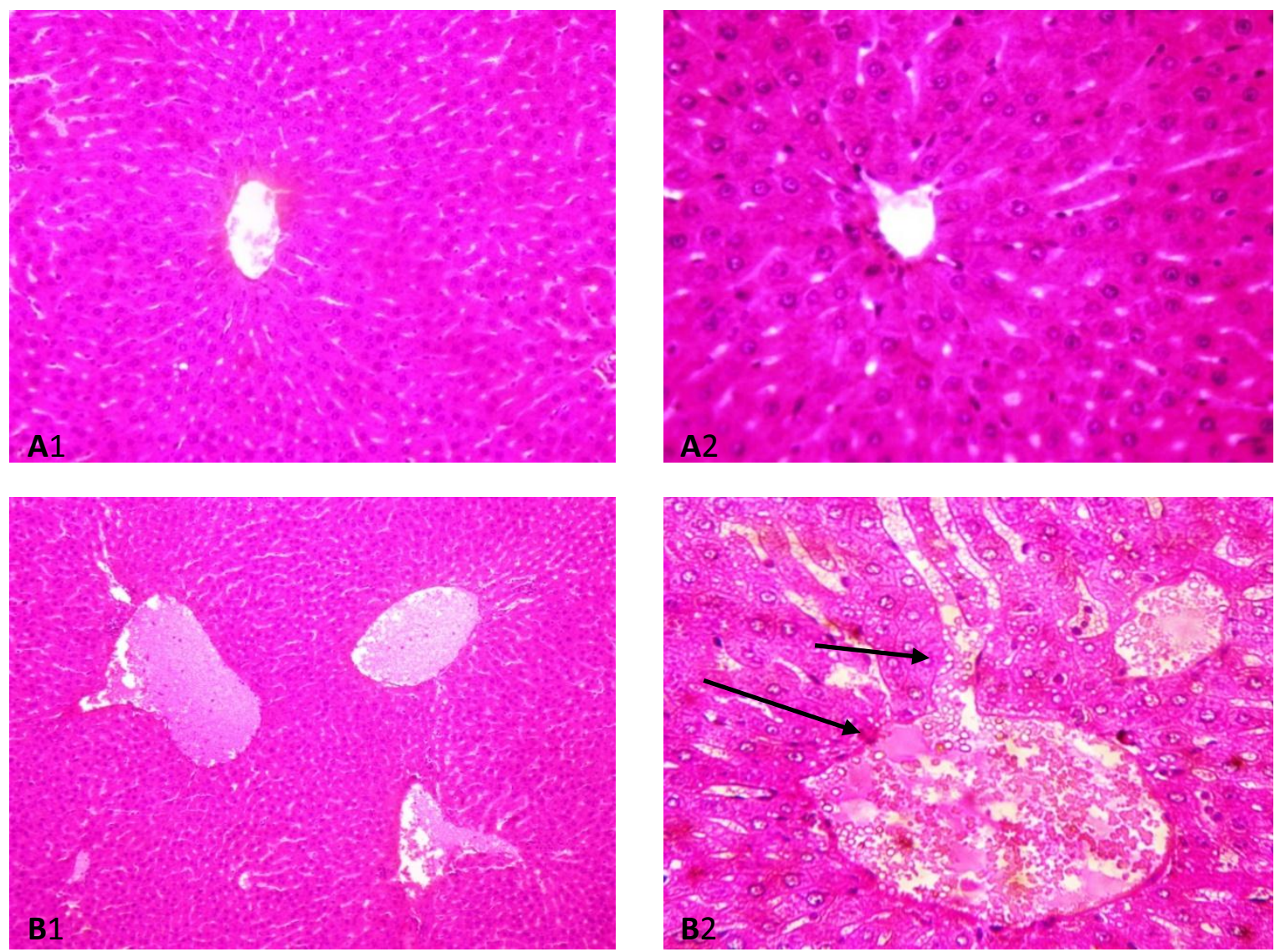

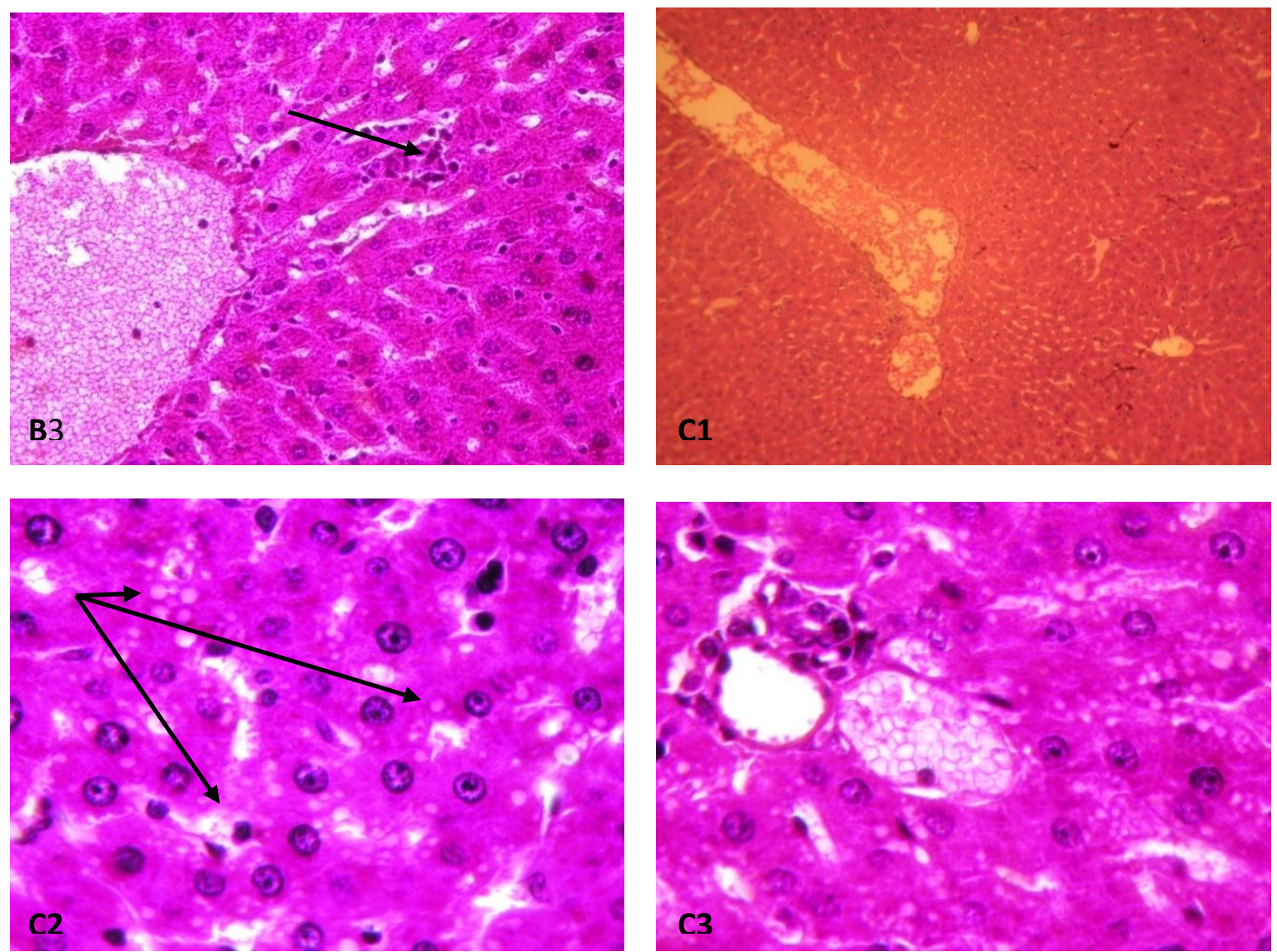

Figure4. Photomicrographs of the liver with the same staining (Hematoxylin-eosin) and different magnification in the control and treated groups. (A1) Micrograph of liver sections of control rat showed the normal trabecular arrangement (H\&E, X250); (A2) with preserved hepatocytes and normal capillaries form (H\&E, X40). (B1) Sections of the liver nicotine-treated rat demonstration marked changes in cellular architecture (H\&E, X100), (B2) congestion of centrilobular veins and sinusoids (H\&E, X400), (B3) and moderate lymphocytic infiltration (Arrows) (H\&E, X400). Sections of the liver nicotine ethanol-treated rat showed worse congestion and dilation of veins, loss of usual concentric arrangement of cells, (C1) varied sizes of hepatocytes with uncertain cellular limits and accumulation of lipid droplets in the cytoplasm (Arrows) (H\&E, X400) and (C2) mild lymphocytic infiltration (Arrows) (H\&E, X400).

\section{Discussion}

Cigarette smoking is common in societies worldwide and has been identified as injurious to human health. The effect of smoking on liver diseases has been studied and these investigations have revealed its harmful effects. ${ }^{46}$ Nicotine, a major toxic component of cigarette smoking ${ }^{47}$ is rapidly absorbed through the lung and is mainly metabolized in the liver. ${ }^{48,49}$ Chronic administration of nicotine in rats reportedly induces cytochrome $\mathrm{P}$ 450 , generates free radicals in tissues and exerts oxidative tissue injury. ${ }^{50,51}$ So, individuals who consume alcohol frequently smoke at the same time ${ }^{52}$ which raises a question regarding the interactive effects of nicotine and ethanol on the antioxidant system in specific tissues of the body. ${ }^{31}$ The liver is a major organ for drug biotransformation, therefore is highly susceptible of the oxidative events associated with the toxicity of nicotine and ethanol. The aim of this study was to evaluate the detrimental effects of chronic administration of nicotine alone or combined with ethanol on the serum biochemical parameters, antioxidant defense status and histopathological changes in liver of male Wistar rats.

The major findings of our work in rats (1) chronically subcutaneous injected nicotine at a dose $2,5 \mathrm{mg} / \mathrm{Kg}$ body weight, for 6 weeks, is associated with an oxidative stress and lipid peroxidation in liver rat; (2) treatment supplementation with ethanol at a dose $2 \mathrm{~g} / \mathrm{Kg}$, enhanced the oxidative stress and the extent of lipid peroxidation in liver tissue; (3) assessment of serum biochemical indicators of liver function reflected cellular injury and alteration of liver function in both treatments, and (4) histopathological examination of the liver tissues revealed marked tissue damage in both treated groups compared to the control. 
In our work, the assessment of serum biochemical parameters revealed a significant increase in LDH level in nicotine-treated animals. LDH, a cytoplasmic marker enzyme is a known indicator of cell and tissue damage by toxic compounds. The increase in LDH activity indicated the cellular damage due to loss functional integrity of cell membranes. In fact, the oxidative tissue injury of hepatic membrane, after chronic exposure to nicotine, produced marked changes in the molecular organization of lipids leading to the increase of membrane permeability and to the leakage of cytoplasmic enzyme such as LDH. Thus, the enhanced LDH activity in nicotine-treated rats can be linked to the increased lipid peroxidation in liver rat.

After chronic administration of nicotine plus ethanol, we observed a significant increase in the activity of indicators of liver function like AST, ALT, ALP and LDH. The increase in the activities of these enzymes in serum was indicative for hepatocyte damage and alteration in liver function. Thus, the enhanced liver damage is due to exacerbated toxicity by combined exposure to nicotine and ethanol.

Taking into account the association of oxidative stress and various pathologic conditions, much attention is being paid on the nicotine effects on oxidative stress in rats long-term submitted to nicotine. In the present study, the profile of antioxidant status in liver after nicotine administration revealed marked alterations in antioxidant enzymes activities. The deficiency of biological antioxidant system is a result of an imbalance between an increase in generation of reactive oxygen species (ROS) and antioxidant levels. These harmful species directly react with a number of biomolecules in cell, including lipids, proteins and nucleic acids, which cause oxidative damage and can ultimately, lead to cells death. ${ }^{53,54}$ The biological antioxidant defense system is an integrated array of enzymes and antioxidants. SOD, which catalyses the dismutation of $\mathrm{O}^{2}-$ to $\mathrm{H}_{2} \mathrm{O}_{2}$ is the first line of antioxidant defense in the body. ${ }^{55}$

The enhanced hepatic SOD activity observed after chronic nicotine administration may be due to increased ROS as a result of nicotine metabolism, which in turn would trigger the activation of SOD activity. Nicotine has been revealed by investigator as individual source of ROS production; reported to be oxidized into its metabolite cotinine mainly in the liver, generate free radicals/ROS in tissues, ${ }^{56}$ and induced oxidative tissue injury. Several mechanisms by which nicotine could generate free radical and promote oxidative stress have been suggested. In fact, nicotine enhances generation of ROS by enhancing the responsiveness of PMN leucocytes to activated $\mathrm{C} 5 \mathrm{a},{ }^{57}$ disrupting of mitochondrial respiratory chain and inducing cytochrome P450 (CYP2A6).

Our study findings showed that exposure to the combination of nicotine plus ethanol significantly increased liver SOD activity. The enhanced hepatic SOD activity indicated an elevated influx of superoxide anions due an additive effect of ethanol. In fact, hepatic ethanol oxidation may enhance generation of ROS by several pathways, via activating xanthine-deshydrogenase / xanthine-oxidase system generating superoxide radical, ${ }^{58}$ microsomal system of ethanol oxidation (MSEO), or via catalase in peroxisomes generating hydrogen peroxide, and especially through the involvement of cytochrome P450IIE1, key enzyme in detoxification of ethanol. ${ }^{59}$

Chronic administration of nicotine increased liver CAT activity. The primary role of CAT is to scavenge $\mathrm{H} 2 \mathrm{O} 2$ that has been generated by free radicals or by SOD in removal of superoxide anions and to convert it to water (Ribiere et al, 1992). The observed increase of hepatic CAT activity may be due to enhanced production of $\mathrm{H}_{2} \mathrm{O}_{2}$.

Chronic administration of nicotine plus ethanol resulted a significant increase in hepatic CAT activity, which may be due an additive effect of ethanol. In fact, CAT has a secondary role in the metabolism of ethanol (Rubin 1993). Therefore, the significant increase in liver CAT activity may be related to excess $\mathrm{H}_{2} \mathrm{O}_{2}$ production resulting from ethanol metabolism or excessive ROS generation due to combined exposure to nicotine and ethanol.

Chronic administration of nicotine significantly increased MDA levels in hepatocytes. Lipid peroxidation can be used as an index for measuring the damage that occurs in membranes tissues as a result of free radical generation. ${ }^{60}$ Thus, the higher level of lipid peroxidation products TBARS in liver nicotine-treated rats in the present study may be due to excessive generation of free radical by nicotine.

After chronic administration of nicotine plus ethanol, we observed an exacerbated increase in MDA content. Thus, the enhanced lipid peroxidation in liver nicotine ethanol-treated rats may be linked to an excessive generation of free radical derived from both metabolism of nicotine and ethanol.

The findings of our study showed adisruption on the activity of antioxidant enzymes and an elevated level of MDA in liver of nicotine-treated rats, indicating the enhanced generation of ROS due to nicotine metabolism and oxidative tissue injury. The increase of lipid peroxidation led to the disruption of the integrity of the membrane of hepatocytes and to the leakage of cytoplasmic enzymes such as LDH.

Moreover, comparing the results of the nicotine-ethanol group to nicotine group, we concluded that the supplementation of ethanol had prejudicial effects on antioxidant system, level of lipid peroxidation, and serum biochemical parameters, more intense than the effects of nicotine alone due to a combined metabolism of nicotine and ethanol.

All these disturbances can be used as indicators for installation and progression of hepatic disease, and confirmed by histopathological analysis. 
In fact, the histopathology of the liver showed a progressive development of the lesions which seems to be due to treatment with nicotine supplemented or not with ethanol. Most liver sections from the nicotine group showed loss of trabecular arrangement, congestion of centrilobular veins and sinusoids, and moderate infiltration of lymphocytes. Supplementation of ethanol increased the histopathological changes in liver tissue byaccumulation of lipid droplets in the cytoplasm accompanied with variable seize of hepatocyte, uncertain cellular limits, worse congestion and dilation of veins and mild mononuclear cell infiltration.

In this investigation, nicotine-treated rats also exhibited significantly increases hematocrit and hemoglobin. These hematological changes are supported by histopathological results which revealed congested blood vessels and inflammatory infiltration.

The hematologic constituents were used as biomarkers for detection of hematotoxic effects of nicotine administration. Several studies have shown that nicotine can induce hematological changes in experimental animals. ${ }^{60,61}$ The increased hematocrit and lymphocytic infiltration may indicate the enhanced of erythropoiesis and activation of the animal's defense mechanisms and immune system. Mountcastle V.B. ${ }^{63}$ has demonstrated that the increase of hematocrit was probably due to contraction of the spleen, which pools red blood cells. Folts and Bonebrake ${ }^{61}$ revealed that nicotine directly administered or through cigarette smoking raises plasma catecholamines by directly stimulating the release of epinephrine from adrenal medulla of the rat. ${ }^{62}$ Thereby, the increase of endogenous epinephrine exacerbated the increase of hematocrit, blood pressure, and heart rate.

However, no significant differences were observed in hematocrit and hemoglobin between the control and nicotine-ethanol groups. These normal values can be linked to hemodilution. Indeed, the nicotine-ethanol group showed a significantly increase $(\mathrm{p}<0.001)$ in consumption of drinking water compared to others experimental groups (data no showed).

In addition, the weight of the liver in the nicotine-ethanol group was significantly higher $(\mathrm{p}<0.005)$ than the liver of control group, that may be due to the fat deposition in cytoplasm of hepatocytes, which lead to suspicion of some degree of toxicity did not occur with nicotine alone. This histopathological change was reminiscent to the formation of fatty liver. It could be due to the increased influx of fatty acids into the liver or to hyperlipidemia. ${ }^{64}$ In addition, it has been reported that the shifting in the redox state and NADH oxidation due to ethanol consumption contribute to hepatic metabolic abnormalities, such as, enhanced hepatic lipogenesis and steatosis. $^{65,66}$

\section{Conclusion}

The findings of the present study gave evidence of hepatic damage caused by chronic nicotine exposure and showed that ethanol supplementation increase the injuries of rat liver. In fact, our data showed that longterm nicotine treatment promoted oxidative stress, increased lipid peroxidation, altered activity of antioxidant enzymes, changed the histological parameters in liver tissue and disrupted biochemical parameters in serum.

The ethanol supplementation during chronic nicotine administration exerted additive detrimental effects on the biochemical parameters of liver function, antioxidant defense system and lipid peroxidation, and aggravated the histopathological alterations in liver tissue by over-production of injurious free radical due to both metabolisms of nicotine and ethanol greater than produced during metabolism of nicotine alone.

\section{References:}

[1]. Baggio, B., Budakovic, A., Gambaro, G., 1998. Cardiovascular risk factors, smoking and kidney function. Nephrol., Dial. Transplant. 7, 2-5.

[2]. Benowitz, N.L., 1988a. Pharmacological aspect of cigarette smoking and nicotine addiction. N.Engl.J.Med. 319, 1318-1330.

[3]. Boyle, P., 1997. Cancer, cigarette smoking and premature death in Europe: a review including the Recommendations of European Cancer Experts Consensus Meeting, Helsinki, October 1996. Lung Cancer. 17, 1- 60.

[4]. Fagerstrom, K., 2002. The epidemiology of smoking: health consequences and benefits of cessation. Drugs. $62,1-9$.

[5]. Goette A., Lendeckel U., Kuchenbecker A., Bukowska A., Peters B., Klein H.U., Huth C., Rocken C., 2007. Cigarette smoking induces atrial fibrosis in humans via nicotine. Heart. 93, 1056-1063.

[6]. Sekhon H., Proskocil B.J., Clark J.A., Spindel E.R., 2004. Prenatal nicotine exposure increases connective tissue expression in foetal monkey pulmonary vessels. Eur. Respir. J. 23, 906-915.

[7]. Zhang, G, Kernan K.A., Thomas A., Collins S., Song Y., Li L., Zhu W., Leboeuf R.C., Eddy A.A., 2009. A novel signaling pathway: fibroblast nicotinic receptor alpha1 binds urokinase and promotes renal fibrosis. J. Biol. Chem. 284, $29050-29064$.

[8]. Schievelbein H., Balfour D.J.K. 1984. Nicotine and the Tobacco Smoking Habit. Pergamon Press, Oxford. 1-15.

[9]. Benowitz, N.L., Porchet, H., Sheiner, L., Jacob, P., 1988b. Nicotine absorption and cardiovascular effects with smokeless tobacco use: Comparison with cigarettes and nicotine gum. Clinical Pharmacology and Therapeutics 4, 23-28.

[10]. Dani, J.A., Heinemann, S., 1996. Molecular and cellular aspects of nicotine abuse. Neuron. 16, 905-908.

[11]. Shivij SB, Camilo A, Moncada AB, Clarkson Jr, Salim M, 2006. Effect of nicotine on lung S-Adenosylmethionine and development of Pneumocystis Pneumonia. J. Biol. Chem. 280(15), 15219-15228.

[12]. Suleyman H, Gumustekin K, Taysi S, Keles S, Oztasan N, Aktas O, et al., 2002. Benifical effects of Hippophaerhamnoides L. on nicotine oxidative stress in rat blood compared with vitamine E. Biol Pharm Bull. 25, 1133-1136.

[13]. Van der Vaar, H., Postma, D.S., Timens, W., 2004. Acute effects of cigarette smoke on inflammation and oxidative stress: a review. Thorax. 713-721.

[14]. Jung BH, Chung BC, Chung S, Shim C, 2001. Different pharmacokinetics of nicotine following intravenous administration of nicotine base and nicotine hydrogen tartarate in rats. J Control Release. 77, 183-190. 
[15]. Kovacic, P., Cooksy, A., 2005. Iminium metabolism for nicotine toxicity and addiction: oxidative stress and electron transfer. Med. Hypotheses. 64, 104-111.

[16]. Ben Ahmed Halima, KhlifiSarra, Rtibi Kais, ElfazaaSalwa and GharbiNajoua. 2010. Indicators of oxidative stress in weanling and pubertal rats following exposure to nicotine via milk. Hum ExpToxicol. 29 (6), 489-496.

[17]. Kalpana C, MenonVP., 2004. Protective effect of curcumin on circulatory lipid peroxidation and antioxidant status during nicotine-induced toxicity. ToxicolMech Methods. 14, 339-343.

[18]. Muthukumaran S., Sdheer AR., MenonVP.,Nalini N., 2008. Protective effect of quercetin on nicotine induced prooxidant and antioxidant imbalance and DNA. Toxicology. 243, 207-215.

[19]. Cashman, J.R., Park, S.B., Yang, Z.C., Wrighton, S.A., Jacob, P., Benowitz, N.L., 1992. Metabolism of nicotine by human liver microsomes: stereoselective formation of trans-nicotineNO-oxide. Chem. Res. Toxicol. 5, 639-646.

[20]. Yildiz D, 2004. Nicotine, its metabolism and an overview of its biological effects. Toxicon. 43, 619-632.

[21]. Wang, S. L.; He, X. Y. \& Hong, J. Y., 2005. Human cytochrome p450 2s1: lack of activity in the metabolic activation of several cigarette smoke carcinogens and in the metabolism of nicotine. Drug Metab. Dispos., 33, 336- 340.

[22]. Altamirano, R. Bataller., 2010. Cigarette smoking and chronic liver diseases. 59, 1159-1162.

[23]. Bataller, R, 2006. Time to ban smoking in patients with chronic liver diseases. Hepatology. 44, 1394-1396.

[24]. Azzalini L., Ferrer E., Ramalho L.N., Moreno M., Dominguez M., Colmenero J., Peinado V.I., Barbera J.A., Arroyo V., Gines P., Caballeria J., Bataller R.,2010. Cigarette smoking exacerbates nonalcoholic fatty liver disease in obese rats, Hepatology .51, $1567-1576$.

[25]. Mori, M., Hara, M., Wada, I., Hara, T., Yamamoto, K., Honda, M., Naramoto, J., 2000. Prospective study of hepatitis B and C viral infections, cigarette smoking, alcohol consumption, and other factors associated with hepatocellular carcinoma risk in Japan. Am. J. Epidemiol. 151,131- 139.

[26]. Yu, M.W., Chiu, Y.H., Yang, S.Y., Santella, R.M., Chern, H.D., Liaw, Y.F., Chen, C.J., 1999. Cytochrome P450 1A1 genetic polymorphisms and risk of hepatocellular carcinoma among chronic hepatitis B carriers. Br. J. Cancer 80, $598-603$.

[27]. Corrao, G., Lepore, A.R., Torchio, P., Valenti, M., Galatola, G., D’Amicis, A., Arico, S., Di Orio, F., 1994. The effect of drinking coffee and smoking cigarettes on the risk of cirrhosis associated with alcohol consumption. A case-control study. Provincial Group for the Study of Chronic Liver Disease. Eur. J. Epidemiol. 10, 657-664.

[28]. Klatsky, A.L., Armstrong, M.A., 1992. Alcohol, smoking, coffee, and cirrhosis. Am. J. Epidemiol. 136, 1248- 1257

[29]. Yuen, S.T., Gogo Jr., A.R., Luck, I.C., Cho, C.H., Ho, J.C.I., Loh, T.T., 1995. The effect of nicotine and its interaction with carbon tetrachloride in the rat liver. Pharmacol. Toxicol. 77, 225-230.

[30]. Gamal H. El-Sokkary, Salvatore Cuzzocrea, Russel J. Reiter, 2007. Effect of chronic nicotine administration on the rat lung and liver: Beneficial role of melatonin Toxicology. 239, 60-67

[31]. Husain K, Scott RB, Reddy KS, Somani SM, 2001. Chronic ethanol and nicotine interaction on rat tissue antioxidant defense system. Alcohol.25, 2:89-97.

[32]. Mehta M.C., Jain A.C., Billie M. 1998. Combined effects of alcohol and nicotine on cardiovascular performance in a canine model. J CardiovascPharmacol. 31, 930-936.

[33]. Wetscher G.J., Bagchi M., Bagchi G., Perdikis G., Hinder P.R., Glaser K., Hinder R.A., 1995. Free radical production in nicotine treated pancreatic tissue.FreeRadicBiol Med.18, 877-882.

[34]. Cahill A, Cunningham C, Adachi M, et al., 2002. Effects of alcohol and oxidative stress on liver pathology: the role of the mitochondrion. Alcoholism ClinExp Res. 907-15.

[35]. Lieber CS. 2000. Alcohol and the liver: metabolism of alcohol and its role in hepatic and extrahepatic diseases. Mt Sinai J Med. 67, 84-89.

[36]. Zima T, Fialova L, Mestek O, et al., 2001. Oxidative stress, metabolism of ethanol and alcohol related diseases. J Biomed Sci. 8, 59-70.

[37]. Anthony, J.C., Echeagaray-Wagner, F., 2000. Epidemiologic analysis of alcohol and tobacco use. Alcohol Res. Health. 24, 201208.

[38]. Falk, D.E., Yi, H.Y., Hiller-Sturmhöfel, S., 2006. An epidemiologic analysis of co-occurring alcohol and tobacco use and disorders: findings from the National Epidemiologic Survey on Alcohol and Related Conditions. Alcohol Res. Health. 29, 162171.

[39]. Giles AR., 1987. Guidelines for the use of animals in biomedical research. ThrombHaemost. 58, $1078-84$.

[40]. Zijlstra W.G., Kampen V.E.1960. Standardization of hemoglobinometry. The

[41]. Yagi K., 1976. A simple fluorometric assay for lipoperoxide in blood plasma. Biochem. Med. 15, $212-216$.

[42]. Misra, H.P., Fridovich, I., 1972. The role of superoxide anion in the autoxidation of epinephrine and a simple assay for superoxide dismutase. J. Biol. 247: 3170-3175.

[43]. Aebi H., 1984. Catalase in vitro. Methods Enzymol. 105, 121-126.

[44]. Bradford M., 1976. A rapid and sensitive for the quantification of microgram quantities of protein utilizing the principle of protein-dye binding. Anal Biochem. 72, 248-251.

[45]. Bancroft J.D. and Stevens A., 1990. Theory and Practice of Histological Techniques, Churchill Livingstone, Edinburgh.

[46]. Takashi H., Masashi Y., Tadahito S., Mieko K. and Akira T, 2003. Intraportal nicotine infusion in rats decreases hepatic blood flow through endothelin-1 and both endothelin A and endothelin B receptors. Toxicology and Applied Pharmacology. 196, 1-10.

[47]. Hofffmann, D., Rivenson, A., Hecht, S.S., 1996. The biological significance of tobacco-specific N-nitrosamines: smoking and adenocarcinoma of the lung. Crit. Rev. Toxicol. 26:199-211.

[48]. V. Pachauri and S.J.S. Flora. 2013. Effect of nicotine pretreatment on arsenic-induced oxidative stress in male Wistar rats. Hum ExpToxicol. 32, 972-982.

[49]. Jaffe, J.H., 1990. Drug addiction and drug abuse. In: Gilman, A.G., Rall, T.W., Niess, A.S., Taylor, P. (Eds.), Goodman and Gillman's the Pharmacological Basis of Therapeutics, 8th ed. Pergamon, New York. 522-573.

[50]. Anandatheerthavarada, H.K., Williams, J.F., Wecker, L., 1993. The chronic administration of nicotine induces cytochrome P-450 in rat brain. J. Neurochem. 60, 1941-1944.

[51]. Guan, Z.Z., Yu, W.F., Nordberg, A., 2003. Dual effects of nicotine on oxidative stress and neuroprotection in PC12 cells. Neurochem. Int. 43: 243-249.

[52]. Hughes J.R., 1993.Treatment of smoking cessation in smokers with past alcohol/drug problems. J Subst Abuse Treat, 10:181-187.

[53]. Ames, B, N., Shigenaga, M.K, \& Hagen, T.M., 1993. Oxidants, antioxidants, and degenerative diseases of aging. ProcNatl SCI USA. 90, 7915-7922.

[54]. McCord, JM., 1993. Human disease, free radicals, and the oxidant/antioxidant balance. ClinBiochem. 26, $351-357$. 
[55]. Husain, K and Somani, S.M., 1998. Interaction of exercise training and chronic ethanol injestion on testicular antioxidant system in rat. J ApplToxicol. 18, 421-429.

[56]. Yildiz, D., Ercal, N., Armstrong, D.W., 1998. Nicotine enantiomers and oxidative stress. Toxicology. 130, $155-165$.

[57]. Yamazaki H, Inoue K, Hashimoto M, Shimada T: Role of CYP2A6 and CYP2B6 in nicotine C-oxidation by human liver microsomes. Arch Toxicol, 1999, 73, 65-70.

[58]. Kato N., Hijikata M.,Ootsuyama Y., Shimotohno K., 1990. Molecular cloning of the human hepatitis C virus genome from Japanese patients with non-A non-B hepatitis. Proceedings of the National Academy of Sciences, U.S.A. 87, 9524-9528.

[59]. Anandatheerthavarada, H. K., Shankar, S. K., Bhamre, S., Boyd, M. R., Song, B. J., \&Ravindranath, V., 1993. Induction of brain cytochrome P-450IIE1 by chronic ethanol treatment. Brain Res 601, 279-285.

[60]. Dianzani, M. U., 1985. Lipid peroxidation in ethanol poisoning: a critical reconsideration. Alcohol. 20, $161-173$.

[61]. Folts J.D. and Bonebrake F.C., 1982. The Effects of Cigarette Smoke and Nicotine on Platelet Thrombus Formation in Stenosed Dog Coronary Arteries: Inhibition with Phentolamine. Circulation. 65:465-470

[62]. Watts D.T., 1960. The effect of nicotine and smoking on the secretion of epinephrine.

[63]. Mouncastle VB., 1974. Medical Physiology. Saint Louis: CV. Mosby. 1372-1398.

[64]. Crouse, J, and Grundy, S.M., 1984. Effects of alcohol on plasma lipoproteins and cholesterol and triglyceride metabolism in man. J.Lip.Res. 25, 486-496.

[65]. Lieber, C.S., 1997. Role of oxidative stress and antioxidant therapy in alcoholic and non alcoholic liver diseases. Adv. Pharmacol. $38,601-628$.

[66]. Seiva F.R., Amauchi J.F., Ribeiro Rocha K.K., BarboseNovelli E.L., 2009. Effects on N-acetylcysteine on alcohol abstinence and alcohol-induced adverse effects in rats. Alcohool 43, 127-135. 